\title{
EFEITOS DO TREINAMENTO COMBINADO SOBRE A FORÇA, RESISTÊNCIA E POTÊNCIA AERÓBICA EM IDOSAS
}

\author{
EFFECTS OF COMBINED TRAINING ON THE STRENGTH, ENDURANCE AND AEROBIC POWER IN \\ THE ELDERLY WOMEN
}

Artigo Original

ORIGINAL ARTICLE

Artículo Original

\author{
EFECTOS DEL ENTRENAMIENTO COMBINADO SOBRE LA FUERZA, RESISTENCIA Y LA POTENCIA AERÓBICA \\ ENMUJERES MAYORES
}

\begin{abstract}
Janesca Mansur Guedes ${ }^{1}$
(Fisioterapeuta)

Marília Guedes Bortoluzzi

(Biomédica)

Luciana Pauline Matte ${ }^{2}$

(Educadora Física)

Ciele Maria de Andrade ${ }^{2}$

(Educadora Física)

Nilciane Coppatti Zulpo²

(Educadora Física)

Vanessa Sebben ${ }^{3}$ (Fisioterapeuta)

Hugo Tourinho Filho ${ }^{4}$

(Educador Físico)

1. Universidade Regional Integrada do Alto Uruguai e das Missões. Campus de Erechim. RS. Brasil.

2. Universidade de Passo Fundo, Passo Fundo. RS. Brasil.

3. Faculdade Meridional. IMED. Passo Fundo. RS. Brasil.

4. Universidade de São Paulo. Escola de Educação Física e Esporte de Ribeirão Preto. Ribeirão Preto. SP. Brasil.
\end{abstract}

\section{Correspondência:}

URI, Erechim. Curso de Fisioterapia.

Av. Sete de Setembro, 1621,

Erechim, RS. Brasil. 99700-000.

janescaguedes@yahoo.com.br

\section{RESUMO}

Introdução: O envelhecimento traz várias modificações, entre as quais o declínio progressivo das funções fisiológicas do organismo. Tanto o treino de força quanto um programa de resistência aeróbica são importantes para evitar o declínio funcional associado à idade. Objetivo: Avaliar os efeitos de um programa de treinamento combinado de força e resistência aeróbica sobre a força de preensão manual, a massa muscular, a resistência e potência aeróbica de idosas. Métodos: Trinta e cinco mulheres $(65,7 \pm 6,68$ anos) foram divididas em três grupos: treinamento combinado (TC; $n=15)$, treinamento de força (TF; $n=10)$ e treinamento aeróbico (TA; $n=10)$. Cada grupo treinou duas vezes por semana durante oito semanas, sendo que o grupo TC teve treinamento de força e aeróbico uma vez por semana. Foi utilizado o teste estatístico ANOVA et de Student, com $p \leq 0,05$. Resultados: Foi possível verificar melhoras significativas $(p \leq 0,05)$ dos valores de $\mathrm{VO}_{2 \text { pico }}$ no momento da determinação do limiar anaeróbico e aumento na força de preensão manual em todos os grupos pesquisados, assim como dos valores de massa muscular do vasto lateral $(p \leq 0,05)$. Conclusão: Os resultados sugerem que um programa de treinamento combinado é tão eficaz para ocasionar aumentos de massa muscular, potência e resistência aeróbica de idosas, quanto o treinamento de resistência aeróbica ou força realizado duas vezes por semana, organizados separadamente.

Descritores: saúde do idoso; força muscular; treinamento de resistência; exercício.

\section{ABSTRACT}

Introduction: Aging brings several modifications, including the progressive decline of physiological functions of the body. Both strength training and an aerobic endurance program are important to avoid functional decline associated with age. Objective: To evaluate the effects of a combined training program of strength and endurance on handgrip strength, muscle mass, endurance and aerobic power of elderly women. Methods: Thirty-five women (65.7 \pm 6.68 years) were divided into three groups: combined training ( $C T, n=15)$, strength training (ST, $n=10)$ and aerobic training (AT, $n=10)$. Each group trained twice a week during eight weeks, and the CT group had strength and aerobic training once a week. We used the ANOVA statistical test and the Student's t test, with $p \leq 0.05$. Results: It was possible to observe significant improvements $(p \leq 0.05)$ for $V_{2}{ }_{2 \max }$ values when determining the anaerobic threshold and increase grip strength in all groups studied, as well as the muscle mass of the vastus lateralis $(p \leq 0.05)$. Conclusion: The results suggest that a combined training program is as much effective to cause increases of muscle mass, strength, and aerobic endurance in elderly women as aerobic endurance or strength training performed twice a week, arranged separately.

Keywords: health of the elderly; muscle strength; resistance training; exercise.

\section{RESUMEN}

Introducción: El envejecimiento trae diversas modificaciones, incluyendo la disminución progresiva de las funciones fisiológicas del organismo. Tanto el entrenamiento de fuerza como un programa de resistencia aeróbica son importantes para prevenir el deterioro funcional asociado a la edad. Objetivo: Evaluar los efectos de un programa de entrenamiento combinado de fuerza y resistencia aeróbica en la fuerza de prensión manual, la masa muscular, la fuerza y la potencia aeróbica de mujeres mayores. Métodos: Treintay cinco mujeres (65,7 $\pm 6,68$ años) se dividieron en tres grupos: entrenamiento combinado ( $E C, n=15)$, entrenamiento de fuerza $(E F, n=10)$ y entrenamiento aeróbico $(E A, n=10)$. Cada grupo ha entrenado dos veces por semana durante ocho semanas, yel grupo de EC ha tenido entrenamiento de fuerza y aeróbico una vez a la semana. Se utilizó el ANOVA y la prueba t de Student, con $p \leq 0,05$. Resultados: Fue posible observar mejoras significativas $(p \leq 0,05)$ de los valores de $\mathrm{VO}_{2 p i c o}$ en el momento de la determinación del umbral anaeróbico y el aumento de la fuerza de prensión manual en todos los grupos estudiados, asícomo los valores de masa muscular del vasto lateral $(p \leq 0,05)$. Conclusión: Los resultados sugieren que un programa de entrenamiento combinado es tan efectivo para ocasionar aumento de la masa muscular, la fuerza y resistencia aeróbica de mujeres mayores, como el entrenamiento de resistencia aeróbica o fuerza realizado dos veces por semana, dispuestos por separado.

Descriptores: salud del anciano; fuerza muscular; entrenamiento de resistencia; ejercicio. 


\section{INTRODUÇÃO}

O envelhecimento traz várias modificações, entre as quais o declínio progressivo das funções fisiológicas do organismo, como a diminuição da força, da massa muscular (sarcopenia), da frequência cardíaca máxima, da tolerância ao exercício, da capacidade aeróbica e o aumento da gordura corporal'.

Os processos fisiológicos que se alteram com a idade podem ser amenizados e até modificados pelo exercício físico. Os exercícios não retardam nem alteram o processo normal de envelhecimento, tampouco aumentam o tempo de vida humana. No entanto, pode-se dizer que retardam ou evitam o avanço de uma doença que ameace a vida, como, as doenças cardiovasculares, que é a principal causa de morte. Nesse sentido, pode-se dizer que sua prática aumenta indiretamente a expectativa de vida².

Os exercícios de resistência aeróbica e de força são essenciais para um envelhecimento saudável. Os exercícios aeróbicos melhoram a capacidade funcional e aumentam a capacidade cardíaca, prevenindo e reduzindo o risco de doenças cardiovasculares. Os exercícios de força melhoram a função muscular, contribuindo para a redução da frequência de quedas. Ambos os exercícios contribuem para meIhoras significantes na densidade óssea, prevenindo a osteoporose e reduzindo o risco de desenvolver o diabetes mellitus tipo 2; evitam o câncer de colo e de mama, além de reduzir a ansiedade e depressão. Assim, este hábito pode reduzir o risco da doença crônica, de mortalidade precoce, ajudando na manutenção da independência e de um envelhecimento com qualidade 3,4

No entanto, quando os programas de treinamento envolvem as duas modalidades (treinamento combinado), resistência aeróbica e força, uma pode interferir no efeito da outra e impedir, dessa forma, o resultado esperado, o que é referido como o "fenômeno de interferência". Em relação ao treinamento combinado (TC), existe uma divergência nas pesquisas no que diz respeito ao "fenômeno da interferência". Alguns estudos relatam que o TC inibe o desenvolvimento de força-7, assim como se observa uma magnitude mais baixa do desenvolvimento da resistência aeróbica quando comparado a um modo de treinamento realizado de forma isolado ${ }^{8,9}$. Em oposição, outros estudos demonstram que o TC não tem nenhum efeito inibitório no desenvolvimento de força e da resistência aeróbica ${ }^{10,11}$. Nesse contexto, o presente estudo apresenta como objetivo avaliar os efeitos de um programa de treinamento combinado de força e resistência aeróbica sobre a força de preensão manual, a massa muscular e a resistência e potência aeróbica de idosas.

\section{MÉTODOS}

Foram incluídas no estudo mulheres idosas (idade igual ou superior a sessenta anos) voluntárias, não ativas fisicamente por um período de no mínimo um mês, com autorização médica para realizar exercício físico. Todas as participantes foram informadas sobre os riscos e benefícios do estudo e assinaram o Termo de Consentimento Livre e Esclarecido, aprovado pelo Comitê de Ética e Pesquisa da Universidade de Passo Fundo, RS, Brasil, sob o número 0161.0.398.000-09. Foram excluídas do estudo as mulheres que apresentaram uma frequência menor que $90 \%$ nos programas de exercícios físicos propostos pelo estudo; mulheres que participaram de outra forma de exercício físico não proposta pela pesquisa; que apresentassem problema cardíaco e musculoesqueléticos e, por isso, durante o estudo pudessem sofrer alguma intercorrência, comprometendo a sua integridade física.

As participantes foram separadas em três grupos de forma aleatória: o grupo de treinamento de resistência aeróbica ( $T A, n=10)$, que realizou caminhada duas vezes na semana; o grupo de treinamento de força ( $T F, n=10)$, que realizou sessões de exercícios resistidos duas vezes na semana; o grupo de treinamento combinado ( $T C, n=15)$, que uma vez por semana realizou TF e uma vez por semana TA. Em relação as características físicas, não se verificou diferença estatisticamente significativa ao se compararem os valores de massa corporal e IMC antes e após a realização dos diferentes programas de treinamento e, também entre os grupos (Tabela 1).

Para a avaliação da força de preensão manual foi utilizado um dinamômetro manual Kratos ${ }^{\circledast}$ (Cotía, São Paulo, Brasil) nos membros dominante e não dominante. A avaliação foi precedida por uma explicação do movimento de preensão. A participante da pesquisa encontrava-se na posição ortostática com o braço ao longo do corpo. Em seguida foi solicitado ao avaliado que apertasse com força o dinamômetro, primeiro com a mão direita e, depois, com a esquerda. Essa sequência foi repetida três vezes, sendo analisada a maior medida. Todas as participantes eram destras.

Para determinar a resistência aeróbica (limiar ventilatório) e potência aeróbica $\left(\mathrm{VO}_{2 \text { pico }}\right)$ foi utilizado um teste do tipo "rampa" (incremento progressivo de carga), realizado em um cicloergômetro Biotec-1800 (CEFISE - Nova Odessa, Brasil) de frenagem mecânica. Previamente a avaliação, foi explicado à participante os procedimento utilizados durante a execução do teste: colocação da máscara, sinais para interrupção, escala de Borg (graduação de 6 a 20 do esforço percebido a cada minuto, a partir do segundo minuto) e sobre a monitorização dos sinais vitais. O teste iniciou com um aquecimento de dois minutos com carga zero, passando a um período de incremento de carga e um período de recuperação ativa na carga zero com duração de 5 min. No período de incrementação foi aplicada uma carga de 15 Watts, e a potência foi aumentada 15 Watts a cada minuto até o ponto em que a participante alcançasse o limiar ventilatório, ou não conseguisse mais manter a velocidade de $22 \mathrm{~km} / \mathrm{h}$. No final de cada fase era anotada a frequência cardíaca e realizado o monitoramento eletrocardiográfico de cinco derivações, com registro da sensação subjetiva de esforço proposta por Borg ${ }^{12}$, e da pressão arterial a cada 3 min, realizada por um avaliador treinado, pelo método auscultatório, utilizando um esfigmomanômetro de coluna de mercúrio e estetoscópio.

Para coleta dos gases foi utilizado um ergoespirômetro (Inbramed, Porto Alegre, Brasil), a determinação do limiar ventilatório (resistência aeróbica) foi realizada com base no comportamento das seguintes variáveis: equivalente ventilatório de oxigênio $\left(V E / \mathrm{NO}_{2}\right)$ e ventilação

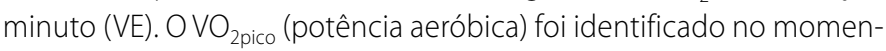
to em que a avaliada fosse incapaz de manter o ritmo estabelecido pelo teste, ou seja, foi o maior valor de $\mathrm{VO}_{2}$ atingido durante o teste de esforço. Foram mensuradas as medidas por meio do analisador de gases (VO2000 ${ }^{\circledR}$ ) (Inbramed, Porto Alegre, Brasil), conectado através de um bucal ${ }^{13}$. Este equipamento para análise de trocas gasosas foi calibrado antes de cada teste de esforço. A sala foi mantida com uma climatização de $22^{\circ} \mathrm{C}$. Os resultados dos testes foram analisados por meio do software Ergo Pc Elite 3.3 ((Micromed ${ }^{\circledR}$, Brasil). Foram utilizados os seguintes critérios para interrupção do teste: frequência cardíaca máxima, aumento ou quedas súbitas da pressão arterial, sensação subjetiva de cansaço, impossibilidade de manter a velocidade de $22 \mathrm{~km} / \mathrm{h}$, alterações eletrocardiográficas ou dos sinais e sintomas.

Tabela 1. Características físicas antes e depois do treinamento.*†

\begin{tabular}{|c|c|c|c|c|c|c|}
\hline & \multicolumn{2}{|c|}{ TF $(n=10)$} & \multicolumn{2}{|c|}{$\mathrm{TA}(n=10)$} & \multicolumn{2}{|c|}{ TC $(n=15)$} \\
\hline & Pré & Pós & Pré & Pós & Pré & Pós \\
\hline Idade (anos) & $67,2 \pm 5,8$ & & $65,9 \pm 6,38$ & & $68,8 \pm 6,1$ & \\
\hline Estatura $(m)$ & $1,59 \pm 0,05$ & & $1,57 \pm 0,06$ & & $1,58 \pm 0,05$ & \\
\hline Peso $(\mathrm{kg})$ & $75,1 \pm 15,2$ & $75,1 \pm 15,25$ & $67 \pm 10,1$ & $63,6 \pm 5,5$ & $67,5 \pm 14,8$ & $69,55 \pm 13,9$ \\
\hline IMC $\left(\mathrm{kg} / \mathrm{m}^{2}\right)$ & $29,7 \pm 5,3$ & $29,7 \pm 5,2$ & $27,03 \pm 3,9$ & $26,08 \pm 1,6$ & $26,8 \pm 4,8$ & $27,4 \pm 4,8$ \\
\hline
\end{tabular}


A área de secção transversal do músculo foi realizada no quadríceps femoral, reto femoral e vasto lateral; foi medida antes e após o programa de treinamento com um varredor ultrassônico composto (GE - PRO Serius - LOGIQ 500) (GE Medical Systems, Milwaukee, Wisconsin, EUA) e um transdutor do corpo convexo $9 \mathrm{MHz}$. A área de secção transversa foi medida no terço distal entre o trocanter maior e a linha lateral do joelho, e calculada pela imagem do sistema computadorizado.

O treinamento de força foi realizado num período de oito semanas, duas vezes por semana e, aproximadamente, com 30 min de duração por sessão, com um intervalo de, no mínimo, 48 horas entre as sessões. Foram realizadas de oito a dez repetições para cada exercício, as cargas foram adequadas na primeira, terceira e sétima semana de treinamento; a velocidade do movimento foi de baixa a moderada intensidade, sempre sob controle; a respiração foi orientada para ser contínua durante cada repetição, expirando ao se levantar a carga e inspirando ao se abaixar a carga ${ }^{14}$. Cada sessão do treinamento foi composta pelos seguintes exercícios: leg press (extensão do quadril e extensão do joelho), mesa extensora (extensão dos joelhos); flexão dos joelhos (com caneleiras na posição ortostática), abdução e adução de quadril.

As participantes deste grupo treinaram resistência aeróbica em dias não consecutivos, duas vezes na semana, por um período de oito semanas. OTA foi realizado por meio de caminhadas em esteira rolante num tempo de 15 a 30 min: nas três primeiras semanas 15 min, na quarta e quinta semanas 20 min, na sexta e sétima semanas 25 min e na oitava semana 30 min. Todas as sessões foram precedidas por cinco min de aquecimento. Para monitorar a intensidade do exercício foi utilizada a escala de Borg e as participantes foram orientadas a permanecer na pontuação 11, que indica a sensação ligeiramente cansativa ${ }^{15}$. A velocidade da esteira para a realização do trabalho aeróbico variou entre 4 e $5 \mathrm{~km} / \mathrm{h}$.

O TC consistiu em TF e TA realizados simultaneamente. As participantes deste grupo treinaram duas vezes na semana em dias não consecutivos, uma vez na semana TF e uma vez na semana TA. Para o exercício de força, os participantes executaram a prescrição do exercício semelhante ao treinamento aplicado ao grupo de TF, com a única diferença de que esse treinamento de força foi conduzido somente uma vez por semana. Para o exercício da resistência aeróbica, os participantes realizaram uma prescrição do exercício baseada nos princípios do treinamento aplicados ao grupo de TA, com a única diferença de que esse treinamento da resistência também foi conduzido somente uma vez por semana.

Para a análise estatística foi utilizado o programa SPSS 10.0. Foi utilizado o teste estatístico ANOVA com post hoc de Tukey para localizar as diferenças entre os grupos e, o teste $t$ de Student para averiguar diferenças antes e depois do programa de exercícios dos grupos, adotando-se um nível de significância $p \leq 0,05$.

\section{RESULTADOS}

Na Tabela 2 é possível observar que as participantes dos grupos TF, TA e TC apresentaram uma melhora estatisticamente significativa ao se comparar os valores de pré e pós-teste para os valores de $\mathrm{VO}_{2 \text { pico }}$ $(p=0,001 ; p=0,042 ; p=0,022)$. A frequência cardíaca no momento da determinação do $\mathrm{VO}_{2 \text { pico aumentou significativamente para os grupos }}$ de TA eTC ( $p=0,004$ e $p=0,009)$, ao passo que para o grupo de TF não foi possível verificar diferenças significativas $(\mathrm{p}=0,177)$. Na potência aeróbica notou-se uma diferença significativa na avaliação inicial entre os grupos de TA com TF $(p=0,011)$ e o grupo TA com TC $(p=0,028)$ e, na avaliação final, diferença entre os grupos TA e TC $(p=0,034)$.

A resistência aeróbica, identificada, no presente estudo, por meio do limiar ventilatório, apresentou uma melhora significativa quando comparada antes e após a realização dos programas de treinamento TF,TA eTC (Tabela 2). A frequência cardíaca no momento do limiar ventilatório aumentou para o grupo de TA eTC com uma diferença estatisticamente significativa, ao passo que para o grupo TF não teve variação significativa. Ao comparar as médias do limiar ventilatório, entre os grupos, observou uma diferença significativa na avaliação final entre o grupo de TA com o grupo TC $(p=0,000)$ e o grupo TA com o grupo TF $(p=0,002)$.

Os resultados da medida da massa muscular (Tabela 2) mostram que o músculo vasto lateral aumentou em todos os grupos, apresentando diferenças significativas (TF - $p=0,042 ; \mathrm{TA}$ - $p=0,015$; $T C$ - $p=0,010$ ), e o reto femoral apresentou diferença estatisticamente significativa para os grupos de TF $(p=0,002)$ e TA $(p=0,039)$. No grupo TC, apesar de se verificar uma tendência de melhora, não foi possível identificar diferenças significativas para o músculo reto femoral. Não foi possível verificar diferença estatisticamente significativa entre os grupos para esta variável.

Na avaliação da força de preensão manual (Tabela 2) todos os grupos tiveram resultados estatisticamente significativos $(p \leq 0,05)$, tanto para a mão direita como para a esquerda. Não foi possível verificar diferença estatisticamente significativa entre os grupos para esta variável.

\section{DISCUSSÃO}

Os resultados do nosso estudo indicam que o $\mathrm{VO}_{2 \text { pico }}$ em todos os grupos tiveram uma melhora significativa entre o pré e pós teste, o que corrobora com Howley e Franks ${ }^{16}$, os quais afirmam que a queda da potência aeróbica pode ser amenizada pela prática de exercício físicos, já que sofre uma redução de $1 \%$ ao ano a partir dos vinte anos. Assim, os indivíduos que realizam exercício físico o decréscimo da potência aeróbica pode ser adiado até pela metade em consequência do envelhecimento ${ }^{17}$.

A literatura aponta que o treinamento aeróbico é a modalidade de exercício mais apropriada para minimizar os efeitos fisiológicos do processo de envelhecimento no condicionamento cardiorrespiratório e que, geralmente, o treinamento de força provoca adaptações no sistema musculoesquelético ${ }^{18}$. No nosso estudo observa-se que o grupo do TF obteve uma melhora significativa no $\mathrm{VO}_{2 \text { pico }}$ pré e pós-intervenção em relação aos demais grupos. O aumento na força muscular, principalmente nas fibras musculares do tipo I, pode contribuir para o teste no cicloergômetro ${ }^{19}$, o que coincide com outros estudo ${ }^{20}$ que demonstraram uma melhora no limiar anaeróbico após um treinamento de força.

Nesse sentido, pesquisas com idosos, observaram que o treinamento de força acarreta adaptações positivas na resistência aeróbica e força muscularr ${ }^{14,21,22}$, e que a melhora na força muscular pode interferir nas

Tabela 2. Valores da média e desvio-padrão das variáveis relacionadas ao desempenho aeróbico; das medidas dos músculos vasto lateral e reto femoral (em milímetros) e força de preensão (em 10.KGF) das participantes por grupo, pré e pós os programas de treinamentos.*

\begin{tabular}{c|c|c|c|c|c|c}
\hline & \multicolumn{2}{|c|}{$T F(\mathbf{n}=\mathbf{1 0})$} & \multicolumn{2}{c|}{ TA $(\mathbf{n}=\mathbf{1 0})$} & \multicolumn{2}{c}{ TC $(\mathbf{n}=15)$} \\
\hline & Pré & Pós & Pré & Pós & Pré & Pós \\
\hline $\mathrm{VO}_{\text {pico }}$ & $17,4 \pm 4,1$ & $23,2 \pm 6 \neq$ & $22,2 \pm 3,2$ & $25,2 \pm 5,3 \neq$ & $18,5 \pm 3,6$ & $20,6 \pm 3,5 \neq$ \\
\hline $\mathrm{LV}$ & $10,2 \pm 2,1$ & $12,1 \pm 1,4 \neq$ & $12,0 \pm 1,6$ & $15,1 \pm 1,5 \neq$ & $10,3 \pm 2,3$ & $12,0 \pm 1,9 \neq$ \\
\hline $\begin{array}{c}\text { Vasto } \\
\text { Lateral }\end{array}$ & $7,8 \pm 3,8$ & $10,3 \pm 7,1 \neq$ & $6,8 \pm 1,3$ & $8,3 \pm 1,7 \neq$ & $7,1 \pm 2,8$ & $9 \pm 2,4 \neq$ \\
\hline $\begin{array}{c}\text { Reto } \\
\text { femoral }\end{array}$ & $11 \pm 5,4$ & $12,7 \pm 5,3 \neq$ & $9,8 \pm 2,3$ & $11,6 \pm 2,6 \neq$ & $10,4 \pm 3,6$ & $11,2 \pm 3,1$ \\
\hline $\begin{array}{c}\text { Mão } \\
\text { direita }\end{array}$ & $1,8 \pm 0,4$ & $2,4 \pm 0,4 \neq$ & $2 \pm 0,6$ & $2,5 \pm 0,9 \neq$ & $2,1 \pm 1$ & $2,5 \pm 0,9 \neq$ \\
\hline $\begin{array}{c}\text { Mão } \\
\text { esquerda }\end{array}$ & $1,7 \pm 0,4$ & $2,4 \pm 0,3 \neq$ & $1,8 \pm 0,5$ & $2,6 \pm 0,9 \neq$ & $2 \pm 0,8$ & $2,4 \pm 1 \neq$ \\
\hline
\end{tabular}

‡ Diferença significativa comparado ao valor pré-treino $(p<0,05)$; ${ }^{\text {TF: }}$ grupo treinamento de força; TA: grupo treinamento aeróbico; TC: grupo treinamento combinado; $\mathrm{VO}_{2 \text { pico: }}$ consumo pico de oxigênio; Lv: limiar ventilatório. 
variáveis aeróbicas ${ }^{14}$. Outro estudo relata que após treinamento de força de nove semanas, melhora no $\mathrm{VO}_{2 \text { pico }}$ em indivíduos idosos ${ }^{23}$. Estudos com nonagenários evidenciou-se que o treinamento de força pode levar a melhor aptidão no transporte de oxigênio e que o aumento da atividade da enzima citrato sintase pode favorecer o metabolismo aeróbico, aumentando a capacidade oxidativa ${ }^{24}$.

Em idosos previamente não treinados parece haver uma maior influência do treinamento de força sobre a melhora da capacidade aeróbica máxima e submáxima ${ }^{21,24}$. É possível que o aumento do $\mathrm{VO}_{\text {2pico }}$ encontrado neste trabalho com somente duas e uma sessão de treinamento semanal possa ter ocorrido em função da periodização dos exercícios que procurou otimizar a intensidade do treinamento.

Estudo semelhante foi desenvolvido por Izquierdo et al. ${ }^{25}$, os quais relataram que no teste do ergoespirômetro a medida da potência de pico em idosos durante o período de 16 semanas nos grupos de TA e TC aumentou 16\% e 18\%, respectivamente, visto que o TF aumentou $10 \%$. Nenhuma diferença significativa foi observada no valor do aumento da carga de trabalho entre oTC e o TA. Os achados principais deste estudo foram que o TC em idosos conduziu a ganhos similares na potência aeróbica quando comparado com o grupo que treinou apenas aeróbico. Já outros estudos encontraram um comprometimento na potência aeróbica do grupo que realizou treinamento combinado quando comparado com os resultados do treinamento aeróbico realizado de forma separada ${ }^{6,8}$

Os resultados do presente estudo confirmam que a combinação de somente uma sessão na semana de treinamento de resistência aeróbica e uma vez na semana de treinamento de força pode ser válida para promover a resistência aeróbica em idosos. Esses achados podem ter uma relevância prática importante para ganho de força e para os programas de treinamento da resistência aeróbica para idosos, tendo em vista que são componentes importantes da aptidão da saúde por contribuem na realização das diversas tarefas da vida diária e, consequentemente, para preservar um estilo de vida independente.

Na resistência aeróbica o grupo TA teve um percentual maior do limiar ventilatório, do tempo e da carga de trabalho no momento do limiar ventilatório. À semelhança do $\mathrm{VO}_{2 \text { pico, o comportamento }}$ do limiar ventilatório que no presente estudo avaliou a resistência aeróbica de idosas apresentou melhoras significativas em todos os grupos, incluindo o TC, fato que afasta a possibilidade do fenômeno da interferência, também para essa variável.

$\mathrm{Na}$ avaliação da massa muscular do vasto lateral durante as oito semanas de treinamento, os grupos TF, TA e TC obtiveram um aumento significativo. Já para o músculo reto femoral o grupo TC não apresentou melhora significativa ao se comparar antes e após a aplicação do programa de treinamento. Na avaliação da área de secção transversal do músculo vasto lateral os achados neste estudo são consistentes com a pesquisa de Izquierdo et al. ${ }^{25}$ e Wood et al. ${ }^{26}$, os quais relataram que em idosos o treinamento combinado foi tão eficaz no condicionamento cardiovascular e na força quanto o treinamento somente de força e aeróbico realizado duas vezes na semana.

A área de secção transversal de um músculo está diretamente associada à força muscular ${ }^{27}$. Desse modo, quando esse aumento acontece na musculatura do quadríceps (grupo muscular importante para a independência funcional dos idosos), consequentemente, o idoso apresenta um ganho maior na força e uma maior independência nas atividades de vida diária ${ }^{28}$.

Este estudo não observou nenhuma diferença entre os grupos TF, TA e TC na área de secção transversal do músculo quadríceps. Nesse sentido, nos idosos previamente não treinados que participam de um programa de treinamento de baixa frequência pode haver um efeito sinérgico de um programa combinado e o treinamento de força pode complementar o treinamento aeróbico, e vice-versa ${ }^{25,29}$

A força de preensão manual nas oito semanas de treinamento aumentou de forma significativa em todos os grupos de treinamento (TF, TA, TC) na mão direita e na mão esquerda. Nenhuma diferença significativa foi observada no valor pré e após o programa de exercício entre os grupos. No estudo de Izquierdo et al. ${ }^{25}$ a força de membro inferior durante oito semanas de treinamento aumentou $27 \%$ no grupo que treinou força; no grupo de treino combinado aumentou 22\% e, para o grupo que somente fez treino aeróbico, 8\%. Nenhuma diferença significativa foi observada no valor do aumento da força entre o grupo de treinamento de força e o de treinamento combinado.

Aceita-se que o treinamento de força pode melhorar o desempenho desta capacidade motora em idosos quando o treinamento for composto por 10-12 repetições, realizados a 50-80\% de 1RM e com uma frequência de 2-3 vezes por semana ${ }^{28,29}$. Essa orientação quanto a intensidade e frequência para oTF vai ao encontro dos resultados do presente estudo, pois com duas vezes na semana o programa de treinamento de força levou a um aumento na força de preensão manual; no entanto, os grupos de treinamento combinado (uma vez na semana treino de força) e aeróbico também apresentaram uma melhora estatisticamente significativa. $\mathrm{O}$ mecanismo responsável para o treinamento aeróbico induzir aumentos na força nos idosos ainda não está compreendido inteiramente ${ }^{29}$

À semelhança do presente estudo, no trabalho conduzido por Izquierdo et al. ${ }^{29}$ que teve como objetivo comparar um grupo de treinamento combinado (uma vez por semana treino de força e uma vez por semana treino aeróbico) com dois grupos, que treinaram duas vezes por semana, um de resistência aeróbica e outro de força foi possível verificar que o treino que combinou força e resistência aeróbica de baixa frequência acarretaram ganhos de força máxima, de potência de carga para músculos extensores da perna e do braço, bem como condicionamento cardiovascular com apenas uma vez por semana e exercícios de força uma vez por semana.

\section{CONCLUSÃO}

Neste estudo o programa de treinamento com duração de oito semanas, que combinou o exercício aeróbico uma vez na semana com o exercício de força também uma vez na semana em idosas, foi eficaz em proporcionar melhoras na força de preensão manual, no aumento da massa muscular do músculo vasto lateral, e na potência e resistência aeróbica.

Da mesma forma os grupos de treinamento de força e o grupo de treinamento aeróbico também apresentaram uma melhora significativa na força de preensão manual, no aumento da massa muscular do músculo vasto lateral e reto femoral, na potência e resistência aeróbica.

Em síntese, os resultados atuais indicam que um programa de treinamento que combinou uma vez por semana exercícios de resistência aeróbica e exercício de força é tão eficaz em proporcionar melhoras na força de preensão manual, massa muscular, potência e resistência aeróbica quanto o treinamento de força e de resistência aeróbica realizados de forma isolada, duas vezes por semana. Foi possível verificar também que a intensidade, frequência e duração utilizada no programa de treinamento do grupo TC permitiu melhoras nas variáveis analisadas, não sendo verificado o fenômeno da interferência no comportamento da força, massa muscular, resistência e potência aeróbica das idosas que participaram do estudo.

Todos os autores declararam não haver qualquer potencial conflito de interesses referente a este artigo. 
CONTRIBUIÇÕES DOS AUTORES: Cada autor contribuiu individual e significantemente para o desenvolvimento do manuscrito. JMG (0000-0001-5595-3333)* e HTF (0000-0001-6517-064x)* participaram de todas as fases, desde a concepção da ideia científica, da redação do artigo e da realização de todas as etapas pesquisa; MGB (0000-0002-5954-4219)*, LPM (0000-0002-8426-8949)*, CMA (0000-0002-4032-8313)*, NCZ (0000-0003-0970-7769)* contribuíram para a avaliação, reavaliação e acompanharam o programa de treinamento das participantes do estudo. VS (0000-0002-6285-6205)* participou da concepção da ideia científica e da redação científica. HTF foi o orientador desta pesquisa. Todos os autores participaram da discussão dos resultados, da revisão e aprovação da versão final do trabalho. *ORCID (Open Researcher and Contributor ID)."

\section{REFERÊNCIAS}

1. Busby-Whitehead J. Exercícios físicos para idosos. In: Gallo JJ, Busby-Whitehead J, Rabins PV, Silliman RA, Murphy JB. Assistência ao idoso: aspectos clínicos do envelhecimento. Rio de Janeiro: Guanabara Koogan, 2001. p.109-113.

2. Hayflick L. Como e por que envelhecemos. Rio de Janeiro: Campus, 1997.

3. Nelson ME, Rejeski WJ, Blair SN, Duncan PW, Judge JO, King AC, et al. Physical activity and public health in older adults: recommendation from the American College of Sports Medicine and American Heart Association. Med Sci Sports Exerc. 2007;39(8):1435-45

4. Ferreira C, Figueiredo MAC. Condicionamento físico: ativação e saúde para mulheres idosas. Rev Bras Ciências Envelhecimento Hum (RBCEH). 2007;4(2):9-22.

5. Hickson RC. Interference of strength development by simultaneously training for strength and endurance. Eur J Appl Physiol Occup Physiol.1980;45(2-3):255-63.

6. Dudley GA, Djamil, R. Incompatibility of endurance and strength-training modes of exercise. J Appl Physiol.1985;59(5):1446-51

7. Häkkinen $\mathrm{K}$, Alen M, Kraemer WJ, Gorostiaga E, Izquierdo M, Rusko H, et al. Neuromuscular adaptations during concurrent strength and endurance training versus strength training. Eur J Appl Physiol. 2003;89(1):42-52.

8. Nelson AG, Arnall DA, Loy SF, Silvester L $\amalg$, Conlee RK. Consequences of combining strength and endurance training regimens. Phys Ther.1990;70(5):287-94.

9. Gravelle BT, Blessing DL. Physiological adaptation in women concurrently training for strength and endurance. J Strength Cond Res. 2000;14:5-13.

10. Sale DG, MacDougall JD, Jacobs I, Garner S. Interaction between concurrent strength and endurance training. J Appl Physiol. 1990;68(1):260-70,

11. Cadore EL, Pinto RS, Pinto SS, Alberton CL, Correa CS, Tartaruga MP, et al. Effects of strength, endurance, and concurrent training on aerobic power and dynamic neuromuscular economy in elderly men. J Strength Cond Res. 2011;25(3):758-66.

12. Ghorayeb N, Dioguardi GS. Tratado de cardiologia do exercício e do esporte. São Paulo: Atheneu, 2007

13. Robergs RA, Roberts SO. Princípios fundamentais de fisiologia do exercício para aptidão, desempenho e saúde. São Paulo: Phorte, 2002

14. Vincent KR, Braith RW, Feldman RA, Kallas HE, Lowenthal DT. Improved cardiorespiratory endurance following 6 months of resistance exercise in elderly men and women. Arch Intern Med. 2002;162(6):673-78.

15. Westcott WL, Baechle T. Treinamento de força para a terceira idade. São Paulo: Manole, 2001
16. Howley ET, Franks BD. Manual do instrutor de condicionamento físico para a saúde. $3^{\text {a }}$ ed. Porto Alegre: Artes Médicas, 2000.

17. Kasch FW, Boyer JL, Van Camp SP, Verity LS, Wallace JP. Effect of exercise on cardiovascular ageing. Age Ageing. 1993;22(1):5-10

18. Tourinho Filho H. Aspectos fisiológicos do envelhecimento: a visão de um filho. In: In: Both A, Barbosa MHS;Benincá CRS, organizadores. Envelhecimento humano: múltiplos olhares. Passo Fundo (RS): UPF;2003.

19. Kura GG. Efeitos de um programa de treinamento que combina hidroginástica e treinamento resistido sobre as respostas hemodinâmicas e metabólicas em idosos. (Master's dissertation). Pontifica Universidade Católica do Rio Grande do Sul, Porto Alegre, 2008.

20. Cider A, Tygesson H, Hedberg M, Seligman L, Wennerblom B, Sunnerhagen KS. Peripheral muscle training in patients with clinical signs of heart failure. Scand J Rehabil Med. 1997;29(2):121-27.

21. Brentano MA. Os efeitos do treinamento de força e do treinamento em circuito na ativação e na força muscular, no consumo máximo de oxigênio e na densidade mineral óssea de mulheres pós-menopáusicas com perda óssea. Dissertação (Mestrado em Ciências do Movimento Humano). Universidade Federal do Rio Grande do Sul, Porto Alegre, 2004.

22. Parker ND, Hunter GR, Treuth MS, Kekes-Szabo T, Kell SH, Weinsier R, et al. Effects of strenght training on cardiovascular responses during a submaximal walk and a weight-loaded walking test in older females. J Cardiopulm Rehabil.1996;16(1):56-62.

23. Hepple RT, Mackinnon SL, Thomas SG, Goodman JM, Plyley MJ. Quantitating the capillary supply and response to resistance training in older men. Pflugers Arch. 1997;433(3):238-44.

24. Frontera WR, Meredith CN, O'Reilly KP, Evans WJ, Strength training and determinants of $\mathrm{VO}_{2 \max }$ in older men. J App Physiol. 1990;68:329-33.

25. Izquierdo M, Ibañez J, Hakkinen K, Kraemer WJ, Larrión JL, Gorostiaga EM. Once weekly combined resistance and cardiovascular training in healthy older men. Med Sci Sports Exerc. 2004;36(3):435-43.

26. Wood RH, Reyes R, Welsch MA, Favaloro-Sabatier J, Sabatier M, Matthew LC, et al. Concurrent cardiovascular and resistance training in healthy older adults. Med Sci Sports Exerc. 2001;33:1751-8.

27. Frontera WR, Hughes VA, Fielding RA, Fiatarone MA, Evans WJ, Roubenoff R. Aging of skeletal muscle: a 12-yr longitudinal study. J App Physiol. 2000;88:1321-26.

28. Izquierdo M, Hakkinen K, Ibañez J, Garrues M, Antón A, Zúñiga A, Larrión JL, et al. Effects of strength training on muscle power and serum hormones in middle-aged and older men. J Appl Physiol. 2001;90(4):1497-507.

29. Izquierdo M, Häkkinen K, Ibáñez J, Kraemer WJ, Gorostiaga EM. Effects of combined resistance and cardiovascular training on strength, power, muscle cross-sectional area, and endurance markers in middle-aged men. Eur J Appl Physiol. 2005;94(1-2):70-5. 